ACTUALIDAD

\title{
Esquemas terapéuticos pediátricos de enteroparasitosis más frecuentes en nuestro país
}

DRA. ISABEL NOEMI HAUCK *

En el tratamiento de las enteroparasitosis existen numerosas drogas útiles para cada parásito. En estos esquemas hemos preferido sugerir los que poseen un éxito terapéutico reconocido y una menor cantidad de efectos secundarios indeseables, para ello hemos reunido no sólo la experiencia nuestra y de colegas nacionales, sino también la de colegas extranjeros.

En esta nota sólo pretendemos facilitar la labor práctica del médico pediatra, y en ella hemos actualizado los esquemas terapéuticos de algunas parasitosis digestivas más frecuentes en nuestros infantes. Por supuesto no son los únicos en vigencia y probablemente con el advenimiento de nuevas drogas más eficaces serán modificados.

De aquí se desprende su revaluación periódica cada 2-3 años.

\section{PROTOZOOS INTESTINALES}

Amibiasis intestinal.

a) Formas crónicas (portador asintomático).

b) Aguda diarreica leve.

Droga de elección: Metronidazol (Bhidroxyelhyl-metil 5 nitroimidazole). Dosis utilizadas en estos momentos: $10-30 \mathrm{mg} / \mathrm{kg} /$ día repartido en 3-4 veces. Tiempo: 7-10 días.

Administración: Oral.

Intolerancia: digestivas (náuseas, vómitos, meteorismo) malestar epigástrico. Puede causar en casos seleccionados leucopenia por inhibición medular. Puede haber molestias de tipo urticarial, disuria. Todos estos efectos secundarios ceden al suprimir la droga. Se sugiere administrarla de preferencia con las comidas y en los más pequeños

\footnotetext{
* Departamento de Medicina Experimental. Sede Oriente. Universidad de Chile.
}

molerlas y disolverlas en manjar o mermelada (con este procedimiento se mejora su tolerancia digestiva).

Marcas Comerciales: Metronidazol F. N., comprimidos orales de $250 \mathrm{mg}$ (20 comprimidos). Metrofemin "Silesia", comprimidos orales de 250 $\mathrm{mg}$ en envases de 20 comprimidos. Venus "Sanitas", comprimidos orales de $200 \mathrm{mg}$ envases de 21 comprimidos.

Droga sustituto: Teclosan (N-N-Bis diclorxaletil-N-N-bis 2 etoxietil 1-4 bis (aminometil) benceno.

Dosis a usar: a) en niños menores de 8 años $150 \mathrm{mg}$ al día divididos en 3 dosis de $50 \mathrm{mg}$.

b) niños 8-14 años $200 \mathrm{mg}$ al día divididos en 3 dosis. dosis.

c) Adultos $300 \mathrm{mg}$ al día administrados en 3

Tiempo a administrar 5 días.

Vía de administración: Oral.

Intolerancia: los efectos secundarios son mínimos y se describen casos de meteorismo o flatulencia.

Marcas comerciales: Falmonox del laboratorio Winthrop, comprimidos orales de $100 \mathrm{mg}$. Cajas de 15 tabletas.

B) Amibiasis diarreica aguda grave.

1) Disentérica.

2) Amibiasis extraintestinales (absceso hepático amibiano).

En estos casos se sugiere administrar 2 drogas antiamibianas combinadas con el fin de evitar la aparición de cepas resistentes. 
Drogas de elección:

1) Metronidazol: $30-40 \mathrm{mg} / \mathrm{kg} /$ día repartidos en 3-4 dosis, tiempo de administración: 10 días. un $89 \%$.

Exito de Metronidazol sólo, en estos casos de

\section{B) Clorhidrato de Emetina.}

Dosis a usar en niños $1 \mathrm{mg} / \mathrm{kg} /$ día sin sobrepasar los $10 \mathrm{mg}$ en cada dosis a inyectar, repartidos en 2 veces al día.

Tiempo de administración: 7 días.

Vía de administración subcutánea profunda $o$ intramuscular.

Tolerancia: Hipotensor por relajar la musculatura lisa, por lo que se aconseja guardar reposo en cama durante su administración.

Digestivas: náuseas, vómitos, diarrea, cólicos abdominales.

Generales: compromiso estado general, cefalea. dades.

Neurológicas: parestesias del cuello y extremi-

Cardiovasculares: alteración de la conducción aurículo ventricular.

Exito de la terapia sola de Emetina en $68 \%$ de los casos.

No repetir una segunda cura hasta 60 días después de la primera por su tardía eliminación y su efecto acumulativo deletéreo.

La combinación de Emetina y Metronidazol obtiene un éxito terapéutico en el $96 \%$ de los casos.

\section{Marcas comerciales:}

Clorhidrato de Emetina "FN" 40 mgx ml-5 amp. Clorhidrato de Emetina "Clin".

Clorhidrato de Emetina "Silesia".

\section{GIARDIASIS}

Droga de elección: Metronidazol.

Dosis a usar en niños: $20-30 \mathrm{mg} / \mathrm{kg} /$ día y repartidos en 2-3 veces por día.

Tiempo a administrar: 7 días:

Vía de administración: oral.

Tolerancia y Marcas Comerciales: descritas anteriormente.

Droga alternativa: Furazolidona.

Dosis utilizadas: $10 \mathrm{mg} / \mathrm{kg} /$ día repartidos en 2-3 veces.

Tiempo de administración: 5-7 días.

Vías de administración oral.
Tolerancia: Buena, por lo breve de la terapia no se describen efectos secundarios de importancia.

Marcas comerciales: Furoxona Laboratorio "Eaton".

1) Comprimidos de $100 \mathrm{mg}$ en envases de 12 comprimidos.

2) Jarabes: envases de $60-120 \mathrm{ml}$. Cada cucharada sopera de $15 \mathrm{ml}$ contiene $50 \mathrm{mg}$.

\section{ISOSPOROSIS}

Tratamiento sintomático y régimen. No se usa quimioterapia. Evoluciona espontáneamente en 1 a 2 meses.

\section{BALANTIDIASIS}

Droga de elección: Tetraciclina.

Dosis: $20 \mathrm{mg} / \mathrm{kg} /$ día, repartidos en 4 tomas con agua.

Vía: oral.

Tiempo de administrar: 8-10 días.

Intolerancia: En niños menores de 6 años se debe ser cuidadoso con su empleo, pues son depositados en dientes y huesos, causando displacia del esmalte y retardo en el crecimiento.

En terreno predispuesto produce disfunción renal y hepática.

Reacciones de fotosensibilidad durante la ingestión de la droga.

Marcas comerciales: Tetraciclina clorhidrato (FN) cápsulas de $250 \mathrm{mg}$ envases conteniendo 16 cápsulas.

Tetraciclina clorhidrato jarabe. Envases de 60 $\mathrm{ml} .5 \mathrm{ml}$ contiene $125 \mathrm{mg}$.

Droga alternativa: Diyodohidroxiquinoleina.

Dosis: $30 \mathrm{mg} / \mathrm{kg} /$ día, repartidos en 3 dosis. Tiempo: 20 días.

Vía: oral.

Tolerancia: en la mayoría de los casos buena, ocasionalmente se describen en pacientes recibiendo dosis terapéuticas, calofríos, fiebre, dermatitis, molestias abdominales, diarreas, cefaleas.

En Japón se describió un caso de neuritis mielóptica subaguda por lo que se la retiró en una oportunidad del comercio. Sin embargo, no se han descrito casos en nuestro país .

Marcas comerciales: Diyodohidroxiquinoleina "FN" comprimidos de $200 \mathrm{mg}$ en envases de 50 comprimidos.

Diodoquin del laboratorio "Raurich" comprimidos de $20 \mathrm{mg}$ en envases de 40-100 comprimidos. 
Drioquilén del laboratorio "Andrómaco" comprimidos de $200 \mathrm{mg}$ en envases de 50 comprimidos.

\section{GUSANOS REDONDOS}

\section{A) Nematelmintos.}

\section{Ascaris lumbricoides}

Droga de elección: citrato de Piperacina.

Vía de administración: oral.

Dosis en niños: $75 \mathrm{mg} / \mathrm{kg} /$ día en una sola dosis en ayunas.

Tiempo a administrar: 2-3 días.

Exito de la curación: en un $100 \%$ de los casos.

Tolerancia: buena, a altas dosis produce toxicodermias y fenómenos neurotóxicos en pacientes con mala función renal, por ser esta la vía de eliminación.

Nombre comercial: Citrato de Piperacina "FN" envases de $120 \mathrm{ml} .5 \mathrm{ml}$ contienen $500 \mathrm{mg}$.

Antiren de "Silesia", envases de $100 \mathrm{ml} .5 \mathrm{ml}$ contienen $500 \mathrm{mg}$.

Bryrel de "Winthrop" envases de 60 y $120 \mathrm{ml}$. $5 \mathrm{ml}$ contienen $500 \mathrm{mg}$.

Noveril del laboratorio "Andrómaco" frascos de $120 \mathrm{ml} .5 \mathrm{ml}$ contienen $500 \mathrm{mg}$.

Piverma del laboratorio" "Bayer" envases de $60 \mathrm{ml} .5 \mathrm{ml}$ contienen $1.000 \mathrm{mg}$.

Droga sustituto: Pamoato de Pyrantel. Pamoato de trans $1,4,5,6$ tetrahidro 1 metil $2-(2(2$ dietiniol) ) vinil pirimidina hidrogenada.

Vía de administración: oral. dosis.

Dosis niños: $11 \mathrm{mg} / \mathrm{kg}$ máximo $1 \mathrm{gr}$ en 1 sola

Tiempo a administrar: 1 día.

Exito de la curación según algunos autores de un $100 \%$.

Tolerancia: Buena. Efectos secundarios: vómitos y diarrea son muy poco frecuentes.

Presentación: Combantrin del laboratorio PFIZER. Frascos de $15 \mathrm{ml} .5 \mathrm{ml}$ contienen $250 \mathrm{mg}$ de Pirantel base.

\section{TRICOCEFALOSIS}

Droga de elección: mebendazole N-metil (5(6)-benzoil-2benzinidazolil Carbamato, $\mathrm{C}_{16} \quad \mathrm{H}_{13}$ $\left.\mathrm{N}_{3}\right)_{3}$.
Vía de administración: oral.

Dosis recomendada idéntica para adultos y niños independiente del peso corporal: $200 \mathrm{mg}$ diarios repartidos en 2 dosis, preferentemente después de almuerzo y comida.

Tiempo a administrar: 3 días.

Exito de la curación en un $98 \%$ de los casos.

Tolerancia: buena. No se conocen hasta el momento reacciones secundarias adversas.

Presentación: Mensole del laboratorio "Andrómaco", comprimidos de $100 \mathrm{mg}$ caja con $6 \mathrm{com}$ primidos.

Esta droga soluciona con su aparición el gran problema del tratamiento de la tricocefalosis. Con su aparición se facilita la erradicación del Trichuris trichiura en casos de afección masiva y también el de los portadores asintomáticos; sobre este último aspecto existen opiniones controvertidas respecto a la posibilidad de tratarlos o no. Personalmente pensamos se debe tratar todo portador aunque sea asintomático, pues constituye un poco de diseminación de la infección al resto de la comunidad.

\section{OXYURIASIS}

Droga de elección: Pamoato de pirvinio.

Dosis $5 \mathrm{mg} / \mathrm{kg} /$ día por 1 vez en una sola dosis en ayunas.

Tiempo de administración: 1 día.

Vía de administración: oral.

Tolerancia: buena, ocasionales molestias digestivas a mayores dosis de las acostumbradas.

Recomendación: por tratarse de una parasitosis extremadamente difusible en la cual todo el grupo familiar esté probablemente infectado, se recomienda:

1) Tratamiento simultáneo de todo el grupo hogareño para lo cual se adjuntan dosis de adultos que son de $300 \mathrm{mg}$ por una vez en ayunas. Dosis total máxima $380 \mathrm{mg}$.

2) Junto a efectuar el tratamiento descrito ha de cambiarse la ropa interior y exterior, la ropa usada durante el período de infección se debe hervir por 15'. Se aconseja el cambio de sábanas o en su defecto su puesta al sol por un período prolongado.

3) Aseo completo de la casa, encerar el piso, sacar el polvo de los zócalos, superficie de muebles, ventanas, paredes, puertas, limpiar tapa W.C., etc. 
Algunos autores para garantizar la mejoría clínica sugieren, repetir el tratamiento a las 2 semanas de efectuado el primero.

\section{Presentación:}

Marcas comerciales:

1) Pamoato de Pirvinio "FN": frascos de $60 \mathrm{ml}$. Este medicamento aporta $50 \mathrm{mg}$ por cucharadita de $5 \mathrm{ml}$.

2) Povan "Parke Davis", frascos de $50 \mathrm{ml} .5 \mathrm{ml}$ contienen $40 \mathrm{mg}$.

3) 'Parvanol "Sanitas", frascos de $50 \mathrm{ml}$ contienen $40 \mathrm{mg}$.

Droga alternativa:

Nombre: Pamoato de Pyrantel.

Vía de administración: oral.

Dosis a administrar: $11 \mathrm{mg} / \mathrm{kg} /$ máximo $1 \mathrm{gr}$ en una sola dosis.

Tiempo a administrar: 1 día.

Tolerancia: buena.

Para presentación ver ascaridiasis.

Se recomienda repetir una segunda cura al cabo de 2 semanas de efectuada la primera.

Usando esta droga deben cumplirse en igual forma las medidas generales recomendadas al usar Pamoato de Pirvinio.

\section{Gusanos planos.}

Teniasis por:

\section{1) Tenia saginata.}

2) Tenia solium.

3) Dipyllobotrium latum.

Estas tres tenias son denominadas lombrices solitarias.

Droga elección: Niclosamida.

Dosis a emplear: niños bajo 2 años, $500 \mathrm{mg}$ dosis total; niños $2-8$ años $1000 \mathrm{mg}$ dosis total; niños 8-14 años $2000 \mathrm{mg}$ dosis total:

Vía a administrar: oral.

Preparación para la terapia:

1) Régimen sin ingestión de alimentos sólidos en la tarde y noche anterior al día del tratamiento.

2) Día del tratamiento $8 \mathrm{AM}$ dar la mitad de la dosis correspondiente a cada caso según edad. Las tabletas deben ser masticadas y deglutidas con agua o té puro.
9 AM.: masticar la segunda mitad de la dosis correspondiente, procediendo del mismố modo antes descrito.

10 AM.: administración de un purgante salino.

La curación es determinada por la salida del escolex, con el objeto de facilitar su búsqueda, se puede indicar una enema evacuante al día anterior al tratamiento.

Con el fin de determinar la cura parasitológica se sugiere enviar la tenia eliminada al laboratorio, para observar si existió salida del escolex.

Tolerancia: Muy buena, pues no se absorbe y no tiene acción irritante sobre la mucosa intestinal. Cabe hacer notar que este medicamento actúa sobre el helminto, pero no sobre los huevos, por lo que se encarece la administración de un purgante salino, con el fin de evitar la digestión de la tenia y la salida de huevos al lumen intestinal. Esto importaría en el caso de T. Solium porque aumentaría el riesgo de cisticercosis.

\section{4) Hymenolepiasis}

Droga de elección: Niclosamida.

Tiempo de administración: 5-7 días.

Vía de administración: oral.

Dosis recomendada para adultos y niños: primer día 2 comprimidos $(1000 \mathrm{mg})$ masticados en ayunas $\mathrm{y}$ deglutidos con agua, en 1 dosis. Desde el segundo día 1 comprimido $(500 \mathrm{mg}$ ) en igual forma hasta completar los días de tratamiento. En caso de persistencia de la infección, se puede repetir éste aumentando la dosis a 2 comprimidos diarios a partir del segundo día hasta completar el período de tratamiento.

Marcas comerciales: Niclosamida "F.N", comprimidos de $500 \mathrm{mg}$. Envases de 4 comprimidos.

Yomesan "Bayer" comprimidos de $500 \mathrm{mg}$, envases de 4 comprimidos.

\section{REFERENCIAS}

1.- Agosin y colaboradores. Acción de la Terramicina sobre el Balantidium coli. Estudio in vitro. Bol. informac. Parasitarias. Vol. 5, 42.43, 1950, Chile.

2.-Perches A., Stoopen M., Segovia E. Estado actual del tratamiento médico de la amibiasis invasora. 59 Seminario sobre amibiasis. Arch. Invest. Med. Supl. 1, 245-256, 1973, México.

3.- Atías A. Algunos esquemas terapéuticos de las parasitosis digestivas. Bol. Chile. Parasit. Vol. 26, 36-43, 1971, Chile. 
4.-Donoso F. y col. Infecciones por Ascaris lumbricoides en niños tratados con Citrato de Piperacina. Bol. Chile. Parasit. Vol. 10. 49-53, Chile.

5.-Drugs for parasitic infection. Chemical Letter on Drugs and Therapeutics. January 18, Vol. 16 No 392: 5-12, USA, 1974.

6.-Fainguenbaum J. Sobre el tratamiento de la amibiasis. Bol. Informaciones parasitarias chilenas. Vol. 5: 33-34 Jul-Sept., 1950.

7.-Goodman L., Gilman A. The Pharmacological basis of therapeutics. Coprighit. The Macmillan Company. III edicion 1971. New York, USA.

8.- Nelson Vaughan, Mac-kay. Tratado de Pediatria. Editorial Salvat. Barcelona, España, 1971.
9.-Landa L., Perches A. León A. Sepúlveda B. El tratamiento del absceso hepático amibiano agudo con Metronidazol. Tercer Seminario sobre amibiasis. Arch. Invest. Med. Supl. 1. 421-426, (México), 1971.

10.- Schenone H. y col. Tratamiento y quimio-profilaxis de las protozoosis intestinales mediante administración de Metronidazol. Bol. Chile. Parasit. Vol. 28: 104-106, 1973, Chile.

11. - Schenone H. y col. Tratamiento de la helmintiasis humanas con mebendazole. Bol. Chile. Parasit, Vol. 29: 2-6, 1974, Chile.

12.-Pessoa-Martina. Parasitología Médica. Nona Ediceo. Río de Janeiro. Editora Guanabara. Koogan S. A., 1974, Brazil. 